\title{
Some Results on the Modelling of Spatial Data
}

\author{
Luca Forlizzi ${ }^{1}$ and Enrico Nardelli ${ }^{1,2}$ \\ 1 Dipartimento di Matematica Pura ed Applicata, Univ. of L'Aquila, Via Vetoio, \\ Coppito, I-67010 L'Aquila, Italia. E-mail: \{forlizzi,nardelli\}@univaq.it \\ 2 Istituto di Analisi dei Sistemi ed Informatica, Consiglio Nazionale delle Ricerche, \\ Viale Manzoni 30, I-00185 Roma, Italia.
}

\begin{abstract}
Formal methods based on the mathematical theory of partially ordered sets (i.e., posets) have been used in the database field for the modelling of spatial data since many years. In particular, the use of the lattice completion (or normal completion) of a poset has been shown by Kainz, Egenhofer and Greasley [13] to be a fundamental technique to build meaningful representations of spatial subdivisions. In fact, they proved that the new elements introduced by the normal completion process can (and have to) be interpreted as being the intersection of poset elements. This is fundamental, from a mathematical point of view, since it means that the lattice resulting from the normal completion is the closure of the given poset with respect to the intersection operation. In this paper we precisely clarify the limitations for the use of lattices as models for spatial subdivisions, by proving sufficient and necessary conditions. Our result gives therefore a sound theoretical basis for the use of lattices built on simplicial complexes as a data model for spatial databases.
\end{abstract}

\section{Introduction}

A class of sets together with a set-containment relation among them models many common situations in spatial databases. For example it may represent a containment relation between geographical objects of the plane or a hierarchical relation between administrative units. The set-containment relation is a partial order relation. Formal methods based on the mathematical theory of partially ordered sets (i.e. posets) have been used for the description of spatial relations since many years 13,819 .

In particular, the use of the lattice completion (or normal completion) of a poset has been shown by Kainz, Egenhofer and Greasley [13 to be a fundamental technique to build meaningful representation of spatial subdivisions. They proposed to represent by means of the elements introduced by the normal completion operator, the set-intersection between sets of the class. Consider for example the class of sets $S$ containing the four sets $A, B, C$, and $D$ shown in Fig. 1.

Each set is represented with an elliptic shape filled with a different pattern. Zones filled with more than one pattern belong to more than one set.

We can represent the class $S$ with the poset $P$ shown in Fig. 2 left. 


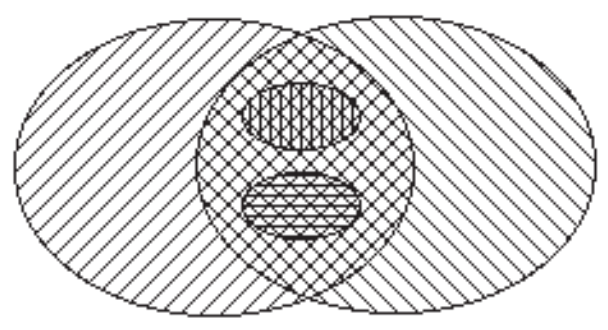
A. C
B $\mathrm{D}$

Fig. 1. A class of sets with a set containment relation
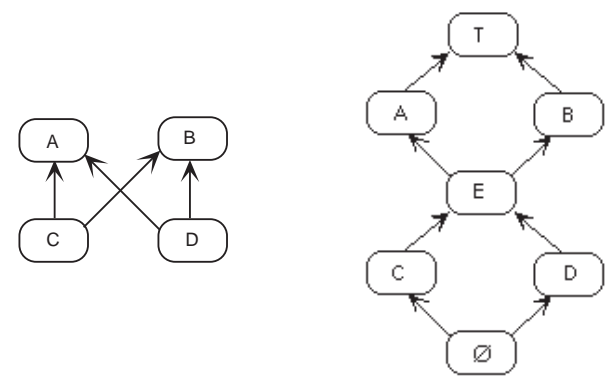

Fig. 2. (left) A poset representation of the class of sets of Fig. 1. (right) The normal completion of the poset in Fig. 2 (left)

Now suppose we want a representation of the closure $S^{\prime}$ of the class $S$ with respect to the set intersection operator (i.e. the class obtained intersecting each possible pair of sets taken from $S$ ). Such a closure is composed by the sets contained in $S$ plus the set $A \cap B$ and the empty set. The set $A \cap B$ is contained in the sets $A$ and $B$ and it contains the sets $C$ and $D$. The normal completion of poset $P$ is the lattice $L$, shown in Fig. 2 right. The lattice $L$ is composed by the elements of $P$ plus a top and bottom element, and a new element labeled $E$, which is smaller than the elements (representing the sets) $A$ and $B$ and greater than those (representing the sets) $C$ and $D$. Therefore, since the relation of set with respect to other sets of $S$ is analogous to that of the element $E$ with respect to other elements of $L$, the lattice $L$ can represent the class of sets $S^{\prime}$, provided that the element $E$ represents the set $A \cap B$.

In the general case, however, using the normal completion operator to represent the set-intersection operator, may lead to incorrect results, as the following example shows. In Fig. 3 a class $S$ of sets with a set containment relation and its closure $S^{\prime}$ with respect to the set intersection operator are represented. 

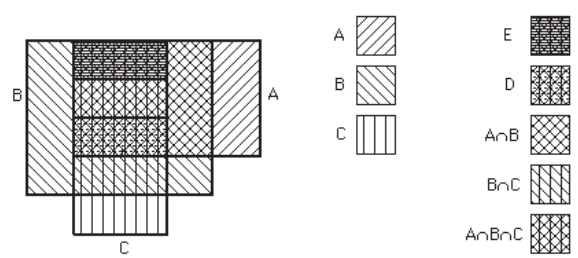

Fig. 3. A class of sets with a set containment relation

The class $S$ is composed by the sets labeled as $A, B, C, D, E, D$ and $T$, where $T \equiv \bigcup_{x \in S} x$ is the greatest set of the class and it is not represented in Fig. 3. As Fig. 3] shows, the class $S^{\prime}$ is composed by the sets contained in $\mathrm{S}$ plus the sets $A \cap B, B \cap C$ and $A \cap C \equiv A \cap B \cap C$. A poset $P$ representing the class $S$ in shown in Fig. 4 left. If we build the normal completion of $P$, we obtain the lattice in Fig. 4 right. The newly created element $X$ is the greatest lower bound of the elements $A$ and $B$, hence it shoud represent the set $A \cap B$. However $X$ is also the greatest lower bound of the elements $B$ and $C$, hence it should represent the set $B \cap C$, but as Fig. 3 shows, $A \cap B$ and $B \cap C$ are different sets and consequently is incorrect to represent them by the same poset element.
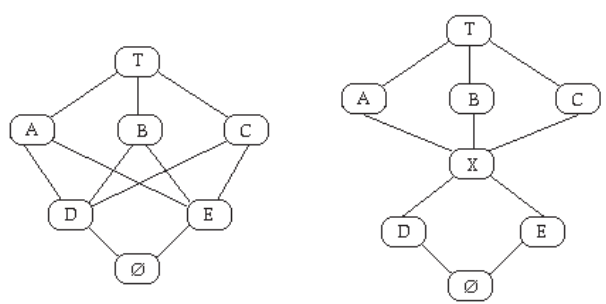

Fig. 4. (left) A poset representation of the class of sets of Fig. 3. (right) The normal completion of the poset in Fig. 4 (left)

In Fig. [5 we see a correct representation of $S^{\prime}$. We have built poset in Fig. 5 starting from Fig. 3 (that shows the class $S$ ) and not from Fig. 4 left (the poset representation of $S$ ). In fact the poset in Fig. 4 left does not provide enough information: for example inspecting Fig. 3 we see that the sets $A \cap C$ and $A \cap B \cap C$ are the same set, but poset in Fig. 4 left cannot carry this information. If $A \cap B \cap C$ was strictly contained in $A \cap C$, see example in Fig. 6, then the poset in Fig. 4 left would still be, without any modification, a representation of this different class.

This fact shows that to represent set intersection operator by means of poset operator we have to provide more information to our representation. A way to do 


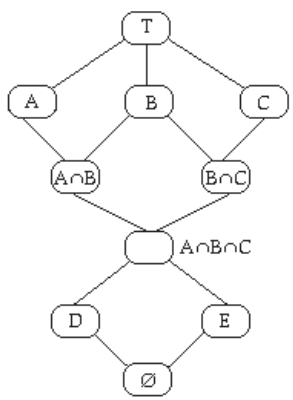

Fig. 5. A representation of the closure with respect to set intersection operator of the class of sets in Fig. [3]
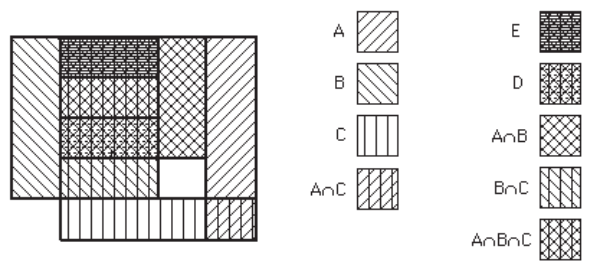

Fig. 6. A class of sets with a set containment relation

this is to include in the class $\mathrm{S}$ a spatial subdivision of the whole domain on which $\mathrm{S}$ is defined. In a poset that represents such a class there is an element for each of the atomic units of the spatial subdivision of $\mathrm{S}$. We show that when a class $\mathrm{S}$ includes a spatial subdivision, the normal completion of its poset representation is a correct representation of $S^{\prime}$. This was the case discussed by Kainz, Egenhofer and Greasley 13 . since they modeled spatial regions by means of simplicial complexes, that include naturally a spatial subdivision.

We highlight in this paper the fundamental role played by the presence of a spatial subdivision for a correct use of the normal completion operator. We give necessary and sufficient conditions for a correct use of lattices as models for spatial relations.

The use of posets as a modelling structure for realities in spatial databases is largely widespread [2]13,8,15,19,11]. Also, a discrete basis for the sets of the class, which is analogous to the universal partition we introduce in Sect. 2, is commonly used in the modelling of geometrical entities [6]10]. Such a discrete basis is indeed the starting point for many efficient data structure based on a space-partitioning criteria, e.g. quadtree [16], grid-file [14], k-d tree [3], cell-tree [9]. Normal completion plays a central role in posets operations 45]. Various studies has been conducted to develop efficient algorithms for its construction. The most interesting, in our opinion, are [12 15[17]. Efficient representation techni- 
ques for posets have been developed in [1,18]. With reference to the use of posets to model spatial databases, in 15 an incremental algorithm to build the normal completion of a poset is given. But the issue of how to interpret the new elements inserted for the completion with respect to the reality of interest is left open.

We close this section with a brief summary of the rest of this paper. In Sect. 2 we introduce formally the definitions of closure of a class of sets with a setcontainment relation with respect to set-intersection, of representation of a class of sets and of universal partition. Section 3 is dedicated to the study of the representation of the closure of a class with respect to set-intersection.

\section{Representations and Closures}

In this section we define formally what we mean by closure of a class of sets with respect to a certain set operator, and what we mean by representation of a class of sets with a set-containment relation by means of a poset. We also introduce in this section the concept of universal partition of a class $S$ with a set-containment relation. It will be used in later sections as a tool to operate efficiently on sets belonging to $S$ and on sets belonging to closures of $S$.

We consider only finite classes, i.e. classes containing a finite number of sets. For technical reasons it is useful to work with classes of sets with a setcontainment relation that contain a greatest set (namely a set that contains every other set of the class) and a least set (namely a set that is contained in every other set of the class). This is not a restriction since if a finite class of sets has not a greatest or a least set, we can always extend it adding respectively the set union of all the sets of the class or the empty set, and then work with the extended class. From now on, when we speak of a class of sets with a setcontainment relation, we always refer to the extended class. All results proved in this section are almost straightforward, hence proofs are omitted.

Definition 1. Let $S$ be a class of sets with a set-containment relation. We define $S^{\cap}$, the closure of $S$ with respect to set-intersection operator, by the following rules:

1. if $s \in S$ then $s \in S^{\cap}$;

2. $\forall s_{1}, s_{2} \in S^{\cap}, s_{1} \cap s_{2} \in S^{\cap}$.

To build correctly the closure of $S$ we need to perform aggregations and subdivisions of sets. For this aim we make use of a universal partition, a subclass of $S$ containing sets that act as building blocks for every other set of $S$ (i.e. every set of $S$ can be obtained applying the set-union operator to a suitable collection of sets of the universal partition).

Definition 2. Let $S$ be a class of sets with a set-containment relation, and let $U_{S} \subseteq S$. We say that $U_{S}$ is a universal partition of $S$ if $\forall r_{1}, r_{2} \in U_{S}$, we have $r_{1} \cap r_{2} \equiv \emptyset$, and $\forall s \in S$ there exist $r_{1}, r_{2} \ldots r_{n} \in U_{S}$ such that $s \equiv \bigcup_{i} r_{i}$.

To associate to each set of the class its building blocks (i.e. the collection of sets of the universal partition that compose the set) we define a mapping. 
Definition 3. Let $S$ be a class of sets with a set-containment relation and a universal partition $U_{S}$. We define the mapping $S_{\text {Base }}: S \mapsto 2^{U_{S}}$ as

$$
S_{\text {Base }}(s)=\left\{r \in U_{S} \mid r \subseteq s\right\} .
$$

The following proposition shows that for each set $\mathrm{s}$ of a class of sets with a set-containment relation, there exists a unique collection of sets of the universal partition whose set-union is equal to s, and that this collection is exactly $S_{\text {Base }}(s)$.

Proposition 1. If $S$ is a class of sets with a set-containment relation and a universal partition $U_{S}$, there exists a unique set $\left\{r_{1}, r_{2} \ldots r_{n}\right\} \in 2^{U_{S}}$ such that $s \equiv \bigcup_{i} r_{i}$. Also $\forall s \in S, s \equiv \bigcup_{r \in S_{\text {Base }}(s)} r$.

The universal partition $U_{S}$ of a class $S$ of sets with a set-containment relation is also a universal partition of $S^{\cap}$.

Corollary 1. Let $S$ be a class of sets with a set-containment relation and a universal partition $U_{S}$. Then $U_{S}$ is a universal partition of $S^{\cap}$.

Thanks to the corollary above, we can apply Definition 3 also to $S^{\cap}$.

Now we define formally what is a representation by means of a poset of a class of sets with a set-containment relation.

Definition 4. Let $S$ be a class of sets with a set-containment relation and let $<P, \leq>$ be a poset. We say that $P$ is a representation of $S$ if there exists an isomorphism between $S$ and $P$.

In the rest of this paper, every time we deal with a representation $P$ of a class $S$ of sets with a set-containment relation, we refer the isomorphism between $S$ and $P$ as $R e p: S \mapsto P$. Of course there exists $R_{e p}{ }^{-1}: P \mapsto S$. Note that since the classes of sets with a set-containment relation we consider have a greatest and a least set, their representations have a greatest and a least element.

In a representation of a class of sets with a set-containment relation and a universal partition, we need to identify the representants of the sets of the universal partition.

Definition 5. Let $S$ be a class of sets with a set-containment relation and a universal partition $U_{S}$, and let $P$ be a representation of $S$. We define universal partition on $P$ the set $U_{P}=\left\{x \in P \mid x=\operatorname{Rep}(r)\right.$ and $\left.r \in U_{S}\right\}$.

Also in the representation we need to refer to representants of the sets of the universal partition whose set-union is a given set. Therefore we introduce the mapping $P_{\text {Base }}($.$) from elements in P$ to subsets of the universal partition defined on $P$.

Definition 6. Let $S$ be a class of sets with a set-containment relation and a universal partition $U_{S}$, and let $P$ be a representation of $S$. For each $p \in P$, we define the mapping $P_{\text {Base }}: P \mapsto 2^{U_{P}}$ as

$$
P_{\text {Base }}(p)=\left\{x \in P \mid x=\operatorname{Rep}(r) \text { and } r \in S_{\text {Base }}\left(\operatorname{Rep}^{-1}(p)\right)\right\} .
$$


In a class of sets with a set-containment relation and a universal partition, a set is 'composed' by sets of the universal partition by means of the set-union operator. In the representation of the class an analogous 'composition' is obtained by means of the $l u b($.$) operator that assign to each subset of a poset its least$ upper bound, as the following theorem shows.

Theorem 1. Let $S$ be a class of sets with a set-containment relation and a universal partition. If $P$ is a representation of $S$ then for each $s \in S$ we have:

$$
\operatorname{Rep}(s)=\operatorname{lub}\left(\left\{y \mid y=\operatorname{Rep}(r) \text { and } r \in S_{\text {Base }}(s)\right\}\right) .
$$

If one thinks to $P_{\text {Base }}($.$) as a mapping between the posets <P, \leq>$ and $<2^{U_{P}}, \subseteq>$, previous proposition translates into the following corollary:

Corollary 2. The mapping $P_{\mathrm{Base}}($.$) is an order embedding from the poset <$ $P, \leq>$ to the poset $<2^{U_{P}}, \subseteq>$.

\section{Representation of Set-Intersection Closure}

\subsection{Introduction}

In this section, given a class $S$ of sets with a set-containment relation and its representation $P$, we study how to derive from $P$ a representation of $S^{\cap}$, the closure of $S$ with respect to the set-intersection operator. Before we proceed with formal investigations on this subject, let us see how the existence of a universal partition modifies the example presented in Fig. 2. In Fig. 7 we show a class $S$ of sets containing five sets $A, B, C, D, E$ which have exactly the same containment relations as the regions in Fig. 2. But the class also contains a universal partition, whose elements coincide with the unit squares of the grid. Sets $A, B, C, D, E$ are shown as aggregations of unit squares identified by different patterns.

A poset representation $P$ for this class of sets is shown in Fig. 8 (the top and the bottom of the poset have been omitted for clarity). We want to construct a representation of $S^{\cap}$, namely a representation which contains also elements that represent sets $A \cap B, B \cap C$ and $A \cap B \cap C$.

Comparing $P$ with the poset in Fig. 4 left we can see that the universal partition provides informations on the class $S$ that were missing in the poset in Fig. 4left. For example elements $1 d, 2 d$ and $3 d$ represent regions contained in both sets $A$ and $B$ but not in set $C$. This fact means that $A \cap B$ and $A \cap$ $B \cap C$ are different sets. Figure 9 shows the normal completion $M(P)$ of poset $P$ (in Fig. 9 also, the top and the bottom of the lattice have been omitted for clarity). Inspecting Fig. 9] (and recalling Fig. 5) we can see that $M(P)$ is a correct representation of class $S^{\cap}$, since elements labeled $X, Y$ and $Z$ represent respectively sets $A \cap B, B \cap C$ and $A \cap B \cap C$. This is a general fact, as we show formally in the following subsection. 

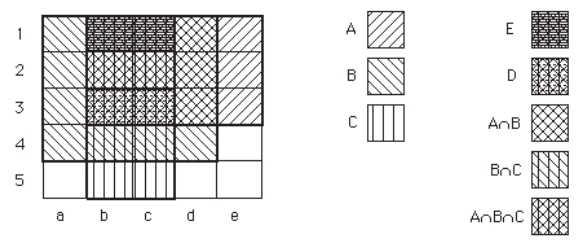

Fig. 7. A class of sets with a set containment relation

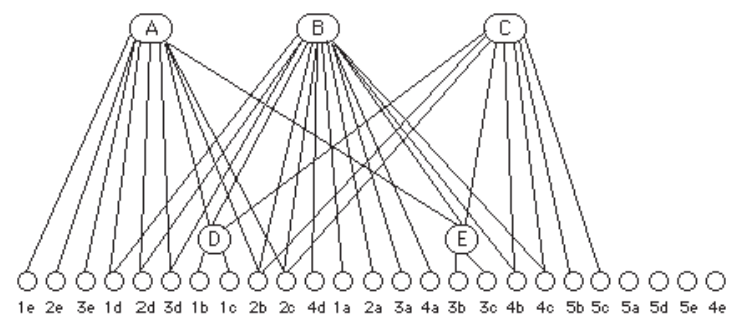

Fig. 8. A poset representation of the class of sets of Fig. 7

\subsection{Sufficient Conditions for Representation of Set-Intersection Closure}

Proofs of results in this section have been omitted since they are either almost straightforward or rather technical. They can be found in the extended version [7. The following theorem tells us that given a representation with a universal partition, the greatest lower bound of the representants of two sets represents, if exists, the intersection between the two sets.

Theorem 2. Let $S$ be a class of sets with a set-containment relation and let $P$ be its representation. Assume $P$ has a universal partition $U_{P}$. For every $x_{1}, x_{2} \in P$,

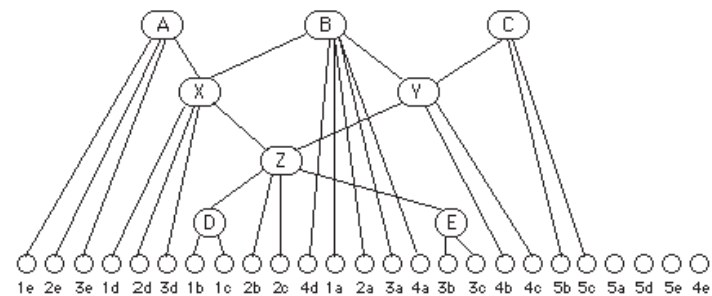

Fig. 9. The normal completion of the poset in Fig. 8 
if there exists $x_{o}=\operatorname{glb}\left(x_{1}, x_{2}\right)$, then

$$
\operatorname{Rep}^{-1}\left(x_{1}\right) \cap \operatorname{Rep}^{-1}\left(x_{2}\right)=\operatorname{Rep}^{-1}\left(x_{o}\right) .
$$

Previous theorem suggests that given a class $S$ of sets with a set-containment relation, in order to provide a representation for the intersection of every subclass of $S$ (i.e. to provide a representation for $S^{\cap}$ ), we need to extend the representation of $S$ to a poset that has a glb for every subset of its elements, namely a lattice. Since the MacNeille completion of a poset to a lattice is the most common way to realize such an extension (and indeed the resulting lattice has interesting properties) we investigate the possibility of representing $S^{\cap}$ by means of $M(P)$, the MacNeille completion of $P$. We prove in the following that if a universal partition of $S$ exists, $M(P)$ is a representation of $S^{\cap}$. Afterwards we discuss what happens if a universal partition does not exist.

In Theorem 3 we will build an isomorphism between the closure of the class $S$ with respect to the set-intersection operation and the normal completion of its representation.

Theorem 3. Let $S$ be a class of sets with a set-containment relation, a universal partition $U_{S}$, and a representation $P$. The mapping IRep $: S^{\cap} \mapsto M(P)$ defined as

$$
\operatorname{IRep}(s)=\left(\left\{g \in P \mid g=\operatorname{Rep}(r), r \in S_{\text {Base }}\right\}^{*}\right)_{*}
$$

is an isomorphism. Hence $M(P)$ is a representation of $S^{\cap}$.

The result of Theorem 3, in the restricted formulation for simplicial complexes, where a universal partition always exists, was proved by Kainz, Egenhofer and Greasley [13. An obvious consequence of Theorem 3 is that $\forall s_{1}, s_{2} \in$ $S, I R e p\left(s_{1} \cap s_{2}\right)=\operatorname{glb}\left(\operatorname{IRep}\left(s_{1}\right), \operatorname{IRep}\left(s_{2}\right)\right)$, namely the representant of the intersection of two sets is the glb of the representants of the sets, as we conjectured in Sect. 1.

\subsection{Necessary Conditions for Representation of Set-Intersection Closure}

Theorem 3 tells us that given a class $S$ of sets with a set-containment relation and its representation $P$, the existence of a universal partition is a sufficient condition for the isomorphism between the posets $\left\langle S^{\cap}, \subseteq>\right.$ and $\langle M(P), \leq>$. Such a condition is not necessary, however, as the example presented in Figs. 2 and 3 shows. In fact in that example, even though there is not a universal partition, we can build the isomorphism by representing the intersection of the sets $A$ and $B$ with the new element $(E)$ introduced in the poset by the MacNeille completion. To find a necessary condition for the isomorphism between the posets $<S^{\cap}, \subseteq>$ and $<M(P), \leq>$, we can proceed in two ways. Either we have to carry out further investigations about the links between the posets $\left\langle S^{\cap}, \subseteq\right\rangle$ and $<M(P), \leq>$ or we have to find additional conditions for the class $S$. We now investigate both alternatives. The following definition introduce a mapping $Z: M(P) \mapsto S^{\cap}$ which we use to show further results for the first alternative. 
Definition 7. Let $S$ be a class of sets with a set-containment relation and let $P$ be a representation of $S$. We define the mapping $Z: M(P) \mapsto S^{\cap}$ as

$$
Z(x)=\bigcap_{y \in(\uparrow x) \varphi(P)} \operatorname{Rep}^{-1}\left(\varphi^{-1}(y)\right) .
$$

The following lemma shows that the mapping $Z($.$) is an order embedding.$

Lemma 1. The mapping $Z: M(P) \mapsto S^{\cap}$ is an order embedding between the posets $<S^{\cap}, \subseteq>$ and $<M(P), \leq>$.

From previous lemma an important result follows immediately.

Lemma 2. Let $S$ be a class of sets with a set-containment relation and a representation $P$. We have $|M(P)| \leq\left|S^{\cap}\right|$, where $M(P)$ is the MacNeille Completion of $P$.

Given the above lemma, a way to find a necessary condition for the existence of an isomorphism between the posets $\left\langle S^{\cap}, \subseteq\right\rangle$ and $\langle M(P), \leq\rangle$ is to find a necessary condition for the sets $S^{\cap}$ and $M(P)$ to have the same cardinality. We achieve this result by means of the mapping $Z($.). The following theorem states a necessary condition for the isomorphism between the posets $\left\langle S^{\cap}, \subseteq>\right.$ and $<M(P), \leq>$.

Theorem 4. Let $S$ be a class of sets with a set-containment relation and a representation $P$. If $S^{\cap}$ is isomorphic to $M(P)$, then $\forall s_{o}, s_{1}, s_{2} \in S$, if $\operatorname{Rep}\left(s_{o}\right)=$ $g l b_{\mathrm{P}}\left(\operatorname{Rep}\left(s_{1}\right), \operatorname{Rep}\left(s_{2}\right)\right)$ then $s_{1} \cap s_{2}=s_{o}$.

Theorem 4 gives a necessary condition for the isomorphism between the posets $<S^{\cap}, \subseteq>$ and $<M(P), \leq>$, namely the fact that $\forall s_{o}, s_{1}, s_{2} \in S$, if $\operatorname{Rep}\left(s_{o}\right)=g l b_{\mathrm{P}}\left(\operatorname{Rep}\left(s_{1}\right), \operatorname{Rep}\left(s_{2}\right)\right)$ then $s_{1} \cap s_{2}=s_{o}$. Note that this condition is not sufficent, as the example of Fig. 3 discussed in Sect. 1 shows. Inspecting Figs. 3 and 4 left we see that $\forall s_{o}, s_{1}, s_{2} \in S$, if $\operatorname{Rep}\left(s_{o}\right)=g l b_{\mathrm{P}}\left(\operatorname{Rep}\left(s_{1}\right), \operatorname{Rep}\left(s_{2}\right)\right)$ then $s_{1} \cap s_{2}=s_{o}$. However posets $\left\langle S^{\cap}, \subseteq>\right.$ and $\langle M(P), \leq>$ are not isomorphic since sets $S^{\cap}$ and $M(P)$ have different cardinalities.

From Theorem 4 the following corollaries follows.

Corollary 3. Let $S$ be a class of sets with a set-containment relation and a representation $P$. If $S^{\cap}$ is isomorphic to the Normal Completion of $P$, then for each $s_{1} \in S_{o}$ and for each $s \in S$ it is $s_{1} \cap s=s_{1}$ or $s_{1} \cap s=\emptyset$, where $S_{o}=\{s \in S \mid \forall x \in S$, if $x \subset s$ then $x=\emptyset\}$.

Corollary 4. Let $S$ be a class of sets with a set-containment relation and a representation $P$. If $S^{\cap}$ is isomorphic to the Normal Completion of $P$, then for every $s_{1}, s_{2} \in S_{o}, s_{1} \cap s_{2}=\emptyset$, where $S_{o}=\{s \in S \mid \forall x \in S$, if $x \subset s$ then $x=\emptyset\}$.

As discussed earlier, the existence of a universal partition is a sufficient, but not necessary condition for the isomorphism between the closure of a class $S$ of sets with respect to the set-intersection operator and the MacNeille comple- 
tion of a representation $P$ of $S$. This means that the converse of Theorem 4 is not true, namely if there exists an isomorphism between $S^{\cap}$ and $M(P)$ not necessarily a universal partition of $S$ exists (see again the example in Figs. 1 and 2). However, thanks to Corollaries 3 and 4, we can effectively pursue the other alternative towards defining necessary conditions for the isomorphism, namely imposing additional constraints to class $S$. For this aim we introduce the following definition.

Definition 8. Let $S$ be a class of sets with a set-containment relation, and let $s_{\mathrm{T}}$ be its greatest set. We say that $S$ is consistent with respect to the set-containment relation if $\bigcup_{x \in S_{o}} x \equiv s_{\mathrm{T}}$, where $S_{o}=\{s \in S \mid \forall x \in S$, if $x \subset s$ then $x=\emptyset\}$.

The assumption of a class of sets to be consistent, is reasonable in many cases, since it means that if a set contains strictly another set, then the difference between the two sets is an 'entity' which has to be represented in the class $S$. For example in a spatial database where a land is represented together with a city contained in it, it seems reasonable that the part of the land outside the city is also identified as an entity.

We can show that for a consistent class $S$ the isomorphism between $S^{\cap}$ and $M(P)$, implies the existence of a universal partition of $S$.

Theorem 5. Let $S$ be a class of sets with a set-containment relation and a representation $P$. If $S^{\cap}$ is isomorphic to the Normal Completion of $P$ and $S$ is consistent, then there exists a universal partition on $S$.

Putting together Theorem 5 and Theorem 3 , we obtain the following corollary that shows how strictly the existence of an isomorphism between $S^{\cap}$ and $M(P)$ is connected with that of a universal partition on $S$.

Corollary 5. Let $S$ be a class of sets with a set-containment relation and a representation $P$. Let $S$ be consistent. Then $S^{\cap}$ is isomorphic to the Normal Completion of $P$ iff there exists a universal partition on $S$.

This result means that in a spatial database that works with poset representations of consistent classes of sets, the only way to perform spatial intersections among sets by means of the normal completion operator, is to provide the database with a universal partition.

\section{Conclusions and Future Works}

Partially ordered sets (posets) are widely used to represent classes of sets with a set containment relation. In this paper we have addressed the problem of how to perform natural set manipulations on a class by means of a poset representation of the class. Concerning set intersection we have stated sufficent and necessary conditions for the correct use of the normal completion operator as a representant of set intersection operator. Moreover, for classes of sets satisfying a little more restrictive condition, we found a condition that is both necessary and sufficient. 
Our results give further motivations to the use of posets to represent classes of sets with a set containment relation, that was first advocated by Kainz, Egenhofer and Greasley in [13], where proved the importance of normal completion as a formal tool in modelling data for spatial databases. Future work will concentrate on characterizing also the set-union operator.

\section{References}

1. H. Aït-Kaci, "A lattice-theoretic approach to computation based on a calculus of partially ordered types", Ph. D. Dissertation, University of Pennsylvania, 1984.

2. E.Apolloni, F.Arcieri, S.Ercoli, E.Nardelli, M.Talamo: "Un modello di riferimento per l'interazione con sistemi per la gestione di dati geografici", (in italian), Convegno Nazionale Sistemi Evoluti per Basi di Dati, Gizzeria Lido, Giugno 1993.

3. J.L.Bentley, "Multidimensional binary search trees used for associate searching", Communications of ACM, 18, 509-517, 1975.

4. G. Birkhoff, "Lattice Theory", American Mathematical Society Colloquium Publications Vol. 25, (Providence, RI: American Mathematical Society), 1967.

5. B.A. Davey, H.A. Priestley, "Introduction to Lattices and Order", Cambridge University Press, 1991.

6. M. Erwing, M. Schneider, "Partition and Conquer", in Spatial Information Theory: A Theoretical Basis for GIS, Vol. 1329 of LNCS, Springer Verlag 1997.

7. L.Forlizzi, E.Nardelli, "On the use of posets as a formal model for spatial data", Technical Report 1/98, Dip. di Matematica, Univ. di L'Aquila, JAN 1998.

8. I. Greasley, "Partially Ordered Sets and Lattices: Correct Metods of Spatial Relations for Land Information Systems", Master's Thesis, University of Maine, Department of Surveying Engineering, Orono, ME, 1990.

9. O.Günther, J.Bilmes, "Tree-based access methods for spatial databases: implementation and performance evaluation", IEEE TKDE, 3(3):342-356, 1991.

10. R.H. Güting, M. Schneider, "Realm-based spatial data types: the ROSE algebra", Fernuniversitat Hagen, Report 141, 1993, VLDB Journal, 4(2), 213-289, April 1995.

11. S.C.Hirtle, "Representational Structures for Cognitive Space: Trees, Ordered Trees and Semi-Lattices", in Spatial Information Theory: A Theoretical Basis for GIS, Vol. 988 of Lecture Notes in Computer Science, Springer Verlag 1995.

12. C. Jard, G.V. Jourdan, J.X. Rampon, "Some online computation of the ideal lattice of posets", IRISA Research Report n.773, 1993.

13. W. Kaintz, M. Egenhofer, I. Greasley, "Modelling spatial relations and operations with partially ordered sets", Int. J. of GIS, vol. 7, no. 3, 215-229., 1993.

14. J. Nievergelt, H.Hinterger, K.C.Sevcik, "The grid file: an adaptable symmetric multikey file structure", ACM TODS, 9(1):38-71, March 1984.

15. L.M. Perry, "Extending (Finite) Partially Ordered Sets to Lattices: An Incremental Approach", Master's Thesis, Univ. of Maine, Dep. of Surv. Eng., Orono, ME, 1990.

16. H. Samet, "The design and analysis of spatial data structures", Addison-Wesley Reading, MA, 1990

17. G. Steiner "An algorithm to generate the ideals of a partial order", Operation Research Letters volume 5 number 6, 1986.

18. M.Talamo, P.Vocca, "An optimal time*space data structure for lattices representation", to be published on SIAM Journal on Computing.

19. M. F. Worboys, "A generic model for planar geographical objects", INT. J. Geographical Information Systems, Vol 6, NO 5, 353-372, 1992. 